\title{
Growth of Silicon Nanowire Arrays Based on Metal-Assisted Etching
}

\author{
Donghee Sihn and Honglae Sohn ${ }^{\dagger}$
}

\begin{abstract}
Single-crystalline silicon nanowire arrays (SiNWAs) using electroless metal-assisted etchings of p-type silicon were successfully fabricated. Ag nanoparticle deposition on silicon wafers in HF solution acted as a localized microelectrochemical redox reaction process in which both anodic and cathodic process took place simultaneously at the silicon surface to give SiNWAs. The growth effect of SiNWs was investigated by changing of etching times. The morphologies of SiNWAs were obtained by SEM observation. Well-aligned nanowire arrays perpendicular to the surface of the silicon substrate were produced. Optical characteristics of SiNWs were measured by FT-IR spectroscopy and indicated that the surface of SiNWs are terminated with hydrogen. The thicknesses and lengths of SiNWs are typically 150-250 $\mathrm{nm}$ and 2 to 5 microns, respectively.
\end{abstract}

Key words: Silicon Nanowires, Metal-assisted Etching, Metal Deposition, Arrays

\section{Introduction}

There is an tremendous interest in the research of silicon nanomaterials for various applications in the fields of opto-electronics, photonics, photovoltaics ${ }^{[1-4]}$, fieldeffect transistors ${ }^{[5-7]}$, optical band pass filters, chemical and biological sensors or drug delivery ${ }^{[8-16]}$. The siliconbased materials are highly favored due to the low production/processing costs, abundance, and high production yields. Since bulk silicon has an indirect badgap, it does not emit visible light. However, the silicon nanomaterials such as quantum dots, nanocrystals, nanowires, and porous silicon have a direct band gap and a quantum confinement effect, they emit visible to infrared light. For the fabrication of the silicon nanowires (SiNWs), many synthetic routs have been developed in recent years. The SiNWs can be fabricated through the metal catalyzed growth, known as chemical vapor deposition or physical vapor deposition methods, or electron beam evaporation.

These techniques often require hazardous silicon precursors, high temperature, complex equipment, and other vigorous conditions even though these methods

Department of Chemistry, Chosun University, Gwangju 501-759, Korea

${ }^{\dagger}$ Corresponding author: hsohn@chosun.ac.kr

(Received : November 12, 2012, Revised : December 15, 2012,

Accepted : December 21, 2012) are quite accessible and well controlled. In addition, the growth of SiNWs is very slow and the coast is high.

An electroless metal deposition (EMD) on a silicon substrate in ionic metal HF solution has been widely used in the microelectronic industries for patterning circuit board, preparing electrode, and making ohmic contacts in the recent years. This technique has an advantage such as simplicity, low coast, and low preparing temperature. EMD which is a localized microelectrochemical redox reaction process has been used for the fabrication of various nanostructures including SiNWs. Both anodic and cathodic process occurs simultaneously at the silicon surface and involves the spontaneous oxidation of silicon atoms. The reduction of metal ions to metallic particles takes place in the absence of an external source of electric current. Pt, Au, $\mathrm{Pd}, \mathrm{Cu}$, and $\mathrm{Ni}$ deposition on silicon wafers have been extensively investigated. The morphologies of SiNWs can be affected by many factors such as the type, doping level, and orientation of the silicon wafer, concentration of $\mathrm{H}_{2} \mathrm{O}_{2}$ in the etchant, etching temperature, and etching time. Herein, we reported the effect of etching times for the growth of SiNWs using metal assistedetching (MAE) based on the metal-seed-induced excessive local oxidation and dissolution of silicon substrates. This method allows the rapid fabrication of high-quality free-standing SiNWs. The length of 
SiNWs could be effectively controlled through tuning the etching time.

\section{Experimental Section}

\subsection{Preparation of SiNWs}

$\mathrm{Si}(100)$ wafers (p-type, boron-doped, $500 \mathrm{~mm}$ thick), was used in this work. $\mathrm{HF}(49 \%), \mathrm{H}_{2} \mathrm{O}_{2}(30 \%), \mathrm{H}_{2} \mathrm{SO}_{4}$ (98\%), and $\mathrm{AgNO}_{3}(>99.9 \%)$ were purchased from Sigma-Aldrich. Silicon wafers were cut into $10 \mathrm{~mm}^{2}$ pieces, which were washed in deionized water, acetone, and ethanol at room temperature to entirely remove contaminated organics from the silicon surface. The wafer pieces were then cleaned in oxidant solution containing $\mathrm{H}_{2} \mathrm{SO}_{4}$ and $\mathrm{H}_{2} \mathrm{O}_{2}$ (4:1) in a volume ratio $4: 1$ for $10 \mathrm{~min}$ under room temperature to form a thin oxide layer and then in a solution of $\mathrm{NH}_{4} \mathrm{OH} / \mathrm{H}_{2} \mathrm{O}_{2} / \mathrm{H}_{2} \mathrm{O}(1$ : 1:5) for $1 \mathrm{~h}$ each. After each cleaning step, the wafer pieces were rinsed with excess deionized water. The cleaned silicon wafer pieces were then immersed in 5\% HF aqueous solution for $3 \mathrm{~min}$ under room temperature and the fresh Si surfaces were H-terminated. The silicon wafers were immediately placed into a $\mathrm{Ag}$ coating solution containing 4.8 M HF and $0.04 \mathrm{M} \mathrm{AgNO}_{3}$, which were slowly stirred for $1 \mathrm{~min}$ under atmosphere ambient. Ag particles were coated on silicon by EMD by immersing silicon pieces in that solution. After a uniform layer of Ag nanoparticles (AgNPs) coating, the silicon substrate was washed with water to remove the extra $\mathrm{Ag}+$ ions and then immersed in oxidizing $\mathrm{HF}$ etching solution composed of $4.8 \mathrm{M} \mathrm{HF}$ and $30 \% \mathrm{H}_{2} \mathrm{O}_{2}$ $(10: 1 \mathrm{v} / \mathrm{v})$ in a reaction vessel. After $1 \mathrm{~h}$ etching in the dark under room temperature, the wafers were washed repeatedly with water and then were immersed in dilute $\mathrm{HNO}_{3}(1: 1 \mathrm{v} / \mathrm{v})$ to dissolve the Ag catalyst. The sample was rinsed with deionized water and dried at room temperature. The wafer surfaces were in deep black, and their back sides in gray. The wafers were washed with $5 \% \mathrm{HF}$ again to remove the oxide layer and then cleaned with water and dried under $\mathrm{N}_{2}$ flow. For the isolation of SiNWs, the prepared SiNW array films were placed in $100 \mathrm{~mL}$ of toluene in a Schlenk flask under argon atmosphere, and then made into particles by ultrasonic fracture in toluene solution for $5 \mathrm{~min}$. After the removal of all removable liquid under a reduced pressure, SiNWs were stored in nitrogen atmosphere prior to use.

\subsection{Instrumentation and Data Acquisition}

FT-IR instrument in the diffuse reflectance mode (Spectra-Tech diffuse reflectance attachment), with diffuse reflectance absorption spectra are reported in absorbance units. Morphologies of SiNWs and SiNWs arrays were observed with a cold field emission scanning electron microscope (FE-SEM, S-4800, Hitachi).

\section{Result and Discussion}

Silver nanoparticles were produced on the surface of silicon wafer using conventional EMD techniques. To investigate the growth of Si NWs, 0.5 and $1 \mathrm{~h}$ of etching time of MAE was investigated. Ag nanoparticles were deposited onto a p-type silicon wafer in $\mathrm{HF} / \mathrm{AgNO}_{3}$ for $1 \mathrm{~min}$. Ag nanoparticles were evenly coated on the surface of silicon wafer and their size was in the range of 10 to $20 \mathrm{~nm}$. Ag nanoparticle-covered Si substrate was immersed in etchant such as $\mathrm{HF} / \mathrm{H}_{2} \mathrm{O}_{2}$ solution at room temperature. Ag-coated silicon wafers were etched in $\mathrm{HF}$ that contained $\mathrm{H}_{2} \mathrm{O}_{2}$ oxidant for 0.5 and $1 \mathrm{~h}$, respectively. The etching of a cleaned silicon wafer proceeds very rapidly when silicon substrates covered with Agnanoparticle films are immersed.

A high-quality and high-oriented SiNWAs were produced on silicon substrates. Fig. 1A is a top view of SEM image of SiNWAs, indicating that SiNWs were aggregated in bundles. Fig. $1 \mathrm{~B}$ is a cross-sectional SEM image of SiNWAs, showing that the length of SiNWs were about 2.5 microns. Fig. $1 \mathrm{C}$ is a cross-sectional SEM image of the SiNWAs at magnification of 20,000 times. Fig. 1D is a side-view SEM image of SiNWAs, indicating the the SiNWs were grown evenly. Fig. 2 showed that the FT-IR spectrum of asprepared SiNW array had a characteristic band centered at 2100 and $900 \mathrm{~cm}^{1}$ for the $v(\mathrm{Si}-\mathrm{H})$ stretching vibration and $\delta(\mathrm{Si}-\mathrm{H})$ bending vibration, indicating that the surface of SiNWs are terminated with hydrogen.

Fig. 3A is a top view of SEM image of SiNWAs which does not show any difference with Fig. 1A. Fig. 3B is a cross-sectional SEM image of SiNWAs, showing that the length of SiNWs were about 7 microns. Fig. $3 \mathrm{C}$ is a cross-sectional SEM image of the SiNWAs at magnification of 10,000 times. Fig. 1D is a side-view SEM image of SiNWAs. These images were almost identical to images in Fig. 1, but the length of 

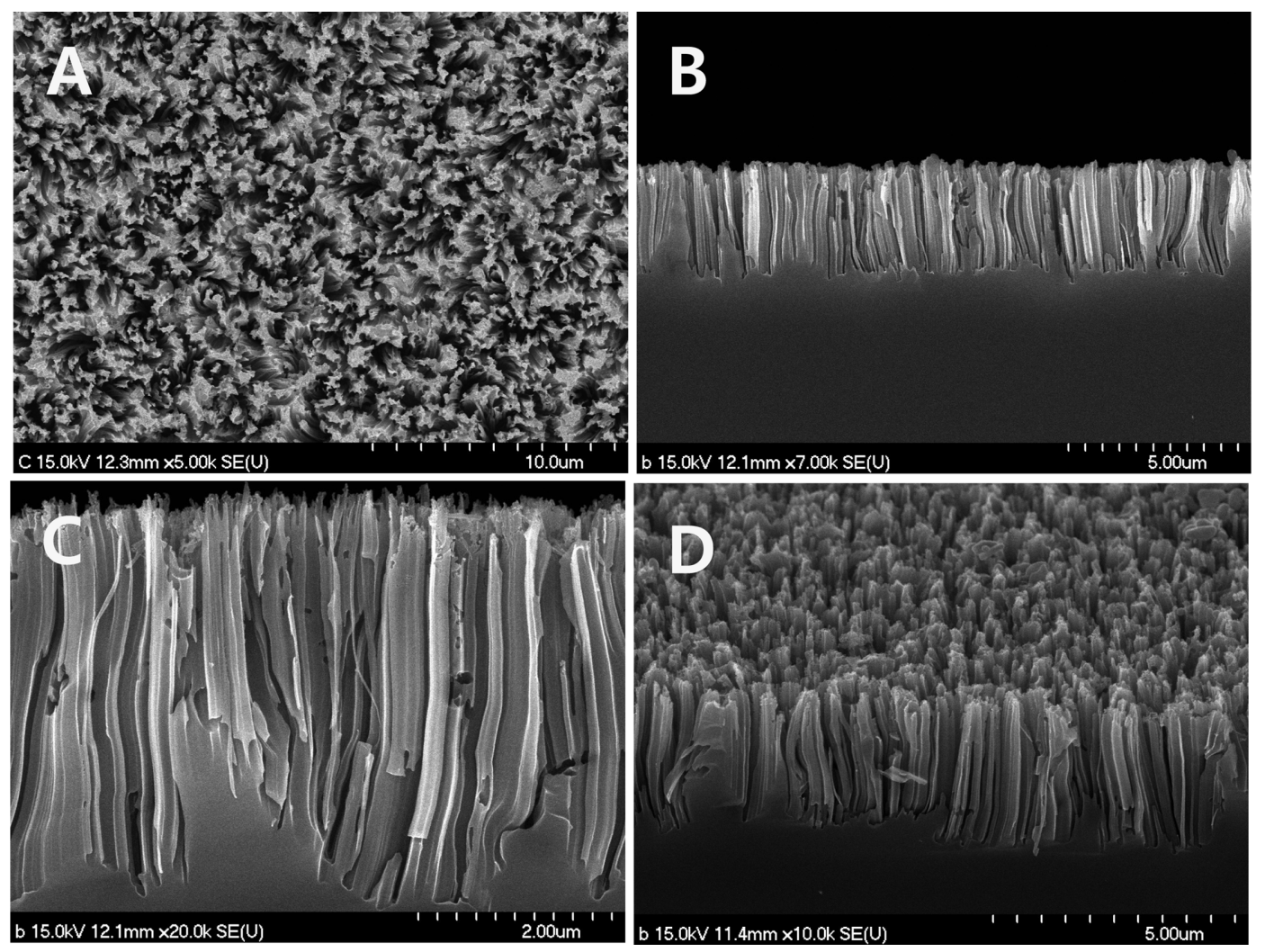

Fig. 1. SEM images of SiNWAs prepared for $30 \mathrm{~min}$. (A) SEM surface image of the SiNWAs, (B) SEM cross-sectional image showing that the length of SiNWs were about 2.5 microns, (C) SEM cross-sectional image of the SiNWAs at magnification of 20,000 times, (D) SEM side-view image of the well-aligned SiNWAs.

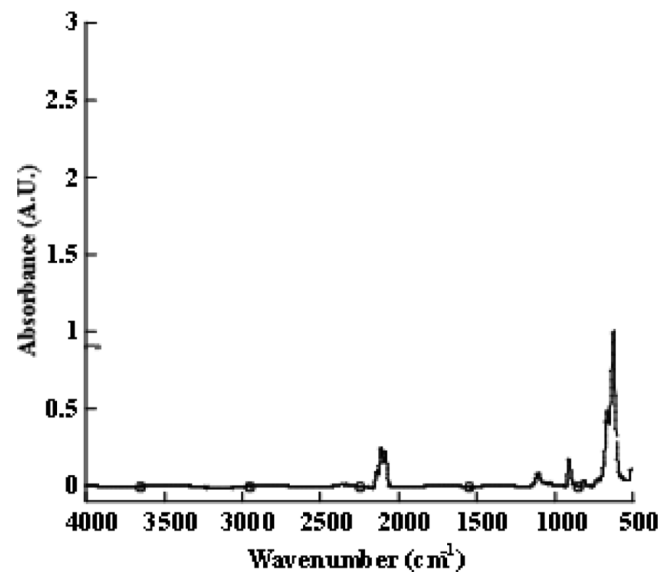

Fig. 2. IR spectrum of SiNWAs.

SiNWs increased without the destruction of any morphology. After the metal assisted-etching in aqueous $\mathrm{HF} / \mathrm{H}_{2} \mathrm{O}_{2}$ solution, large-area aligned SiNWAs perpen- dicular to the silicon surface were produced. The thickness of the SiNWs is about 100 to $200 \mathrm{~nm}$. The length of SiNWs gradually increased with the increase of an etching time. The thicknesses of SiNWs are almost no diameter modulation along the length. With an etching condition of $4.8 \mathrm{M} \mathrm{HF}$ and $30 \% \mathrm{H}_{2} \mathrm{O}_{2}$ etchant solution, the etching rate was about $100 \mathrm{~nm}$ per 10 min. The color of SiNWAs sample is black and SiNWAs did not show any photoluminescence. For the fabrication of SiNWs, p-type silicon wafer showed the best result. The reflection spectrum using white light as an incident light source did not show any significant feature.

Fig. 4 showed the sinking behavior of the Ag nanoparticles. The pore size resulted from the Ag nanoparticles is about 100 to $200 \mathrm{~nm}$. The shapes of SiNWs are both nano-wires and nano-rods. Most of the silicon nanostructures are nano-rods. However, very thin silicon nano-wires were present in the SiNWAs matrix. 

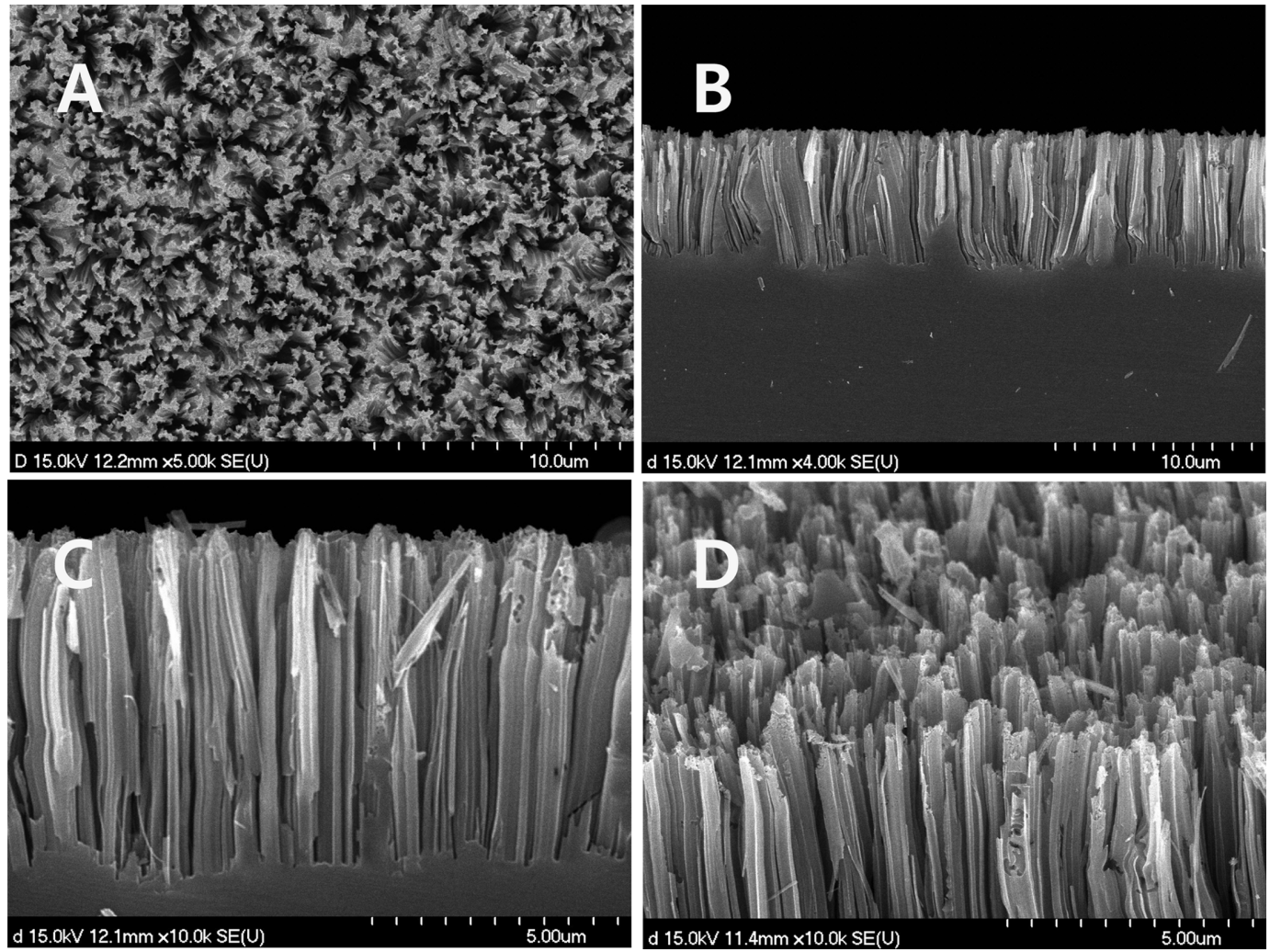

Fig. 3. SEM images of SiNWAs prepared for $1 \mathrm{~h}$. (A) SEM surface image of the SiNWAs, (B) SEM cross-sectional image showing that the length of SiNWs were about 7 microns, (C) SEM cross-sectional image of the SiNWAs at magnification of 10,000 times, (D) SEM side-view image of the well-aligned SiNWAs.

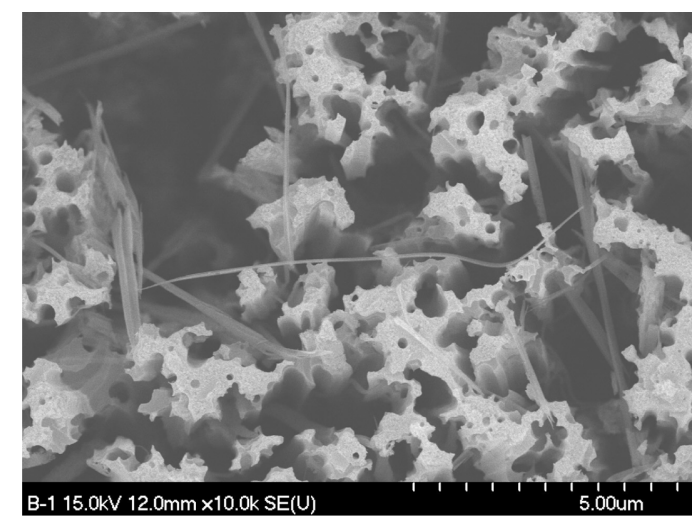

Fig. 4. SEM images of SiNWs showing silicon nanowires.

\section{Conclusion}

A high-quality and high-oriented SiNWAs were produced on silicon substrates. SEM image of SiNWAs indicated that the SiNWs were grown evenly. The thick- ness of the SiNWs is about 100 to $200 \mathrm{~nm}$. The length of SiNWs gradually increased with the increase of an etching time. The etching rate was about $100 \mathrm{~nm}$ per $10 \mathrm{~min}$. The color of SiNWAs sample is black and SiNWAs did not show any photoluminescence. The shapes of SiNWs are both nano-wires and nano-rods. The silicon nanowire arrays formed perpendicular to the silicon surface.

\section{Acknowledgment}

This research was supported by the Basic Science Research Program through the National Research Foundation of Korea (NRF) funded by the Ministry of Education, Science and Technology (2010-0010850).

\section{References}

[1] A. I. Hochbaum, R. Fan, R. He, and P. Yang, "Con- 
trolled growth of Si nanowire arrays for device integration", Nano Lett. Vol. 5, pp. 457-460, 2005.

[2] V. Schmidt, S. Senz, and U. Gösele, "Diameterdependent growth direction of epitaxial silicon nanowires", Nano Lett. Vol. 5, pp. 931-935, 2005.

[3] X. Duan, Y. Huang, Y. Cui, J. Wang, and C. M. Lieber, "Indium phosphide nanowires as building blocks for nanoscale electronic and optoelectronic devices", Nature Vol. 409, pp. 66-69, 2001.

[4] M. D. Kelzenberg, D. B. Turner-Evans, B. M. Kayes, M. A. Filler, M. C. Putnam, N. S. Lewis, and H. A. Atwater, "Photovoltaic measurements in single-nanowire silicon solar cells", Nano Lett. Vol. 8, pp. 710-714, 2008.

[5] S.-M. Koo, Q. Li, M. D. Edelstein, C. A. Richter, and E. M. Vogel, "Enhanced channel modulation in dual-gated silicon nanowire transistors", Nano Lett. Vol. 5, pp. 2519-2523, 2005.

[6] Y. Cui, Z. H. Zhong, D. L. Wang, W. U. Wang, and C. M. Lieber, "High performance silicon nanowire field effect transistors", Nano Lett. Vol. 3, pp. 149152, 2003.

[7] D. H. Jung, "Biosensor based on distributed bragg reflector photonic crystals for the detection of protein A", J. Chosun Natural Sci., Vol. 3, No. 1, p. 33, 2010.

[8] S. H. Jang, "Chemical and Physical Properties of Porous Silicon", J. Chosun Natural Sci., Vol. 4, No. 1, p. 1, 2011.

[9] S. G. Kim, "Optical characterization of smart dust based on photonic crystals and its sensing applications", J. Chosun Natural Sci, Vol. 4, No. 1, p. 7, 2011.

[10] Y. D. Koh, "1-D Photonic crystals based on bragg structure for sensing and drug delivery applications", J. Chosun Natural Sci., Vol. 4, No. 1, p. 11, 2011.

[11] K. S. Jung, "Fabrication and characterization of DBR porous silicon chip for the detection of chemical nerve agents", J. Chosun Natural Sci, Vol. 3, No. 4, p. 237, 2010.

[12] S. D. Cho, "Detection of nitroaromatic compounds with functionalized porous silicon using quenching photoluminescence", J. Chosun Natural Sci., Vol. 3, No. 4, p. 202, 2010.

[13] S. H. Jang, "Study on thickness of porous silicon layer according to the various anodization times", J. Chosun Natural Sci., Vol. 3, No. 4, p. 206, 2010.

[14] S. H. Jang, "Investigation of relationship between etch current and morphology and porosity of porous silicon", J. Chosun Natural Sci., Vol. 3, No. 4, p. 120, 2010.

[15] J. M. Han, "Photoluminescence of Porous Silicon According to Various Etching Times and Various Applied Current Densities", J. Chosun Natural Sci., Vol. 3, No. 3, p. 148, 2010.

[16] Y. D. Koh, "Analysis on Oxidation of Porous Silica Obtained from Thermal Oxidation of Porous Silicon”, J. Chosun Natural Sci, Vol. 3, No. 3, p. 153, 2010. 